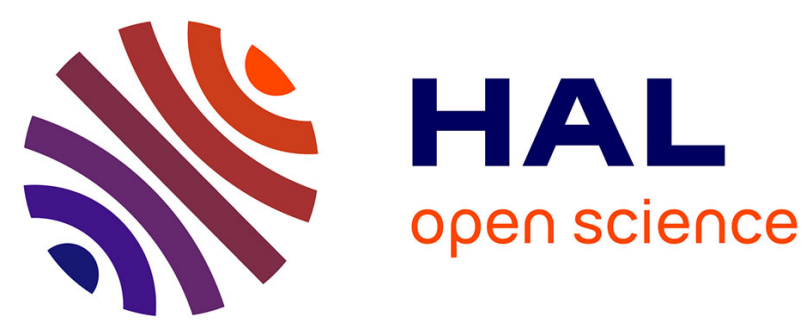

\title{
Mid-infrared multispectral lensless imaging for wide-field and label-free microbial identification
}

Joel Legaludec, Mathieu Dupoy, Véronique Rebuffel, Pierre Marcoux

\section{To cite this version:}

Joel Legaludec, Mathieu Dupoy, Véronique Rebuffel, Pierre Marcoux. Mid-infrared multispectral lensless imaging for wide-field and label-free microbial identification. Proceedings of SPIE, the International Society for Optical Engineering, 2020, 11359, pp.113590B. 10.1117/12.2557502 . cea-03188576

\section{HAL Id: cea-03188576 https://hal-cea.archives-ouvertes.fr/cea-03188576}

Submitted on 2 Apr 2021

HAL is a multi-disciplinary open access archive for the deposit and dissemination of scientific research documents, whether they are published or not. The documents may come from teaching and research institutions in France or abroad, or from public or private research centers.
L'archive ouverte pluridisciplinaire HAL, est destinée au dépôt et à la diffusion de documents scientifiques de niveau recherche, publiés ou non, émanant des établissements d'enseignement et de recherche français ou étrangers, des laboratoires publics ou privés. 


\section{Mid-infrared multispectral lensless imaging for wide-field and label-free microbial identification}

Le galudec, Joël, Dupoy, Mathieu, Rebuffel, Véronique, Marcoux, Pierre

Joël Le galudec, Mathieu Dupoy, Véronique Rebuffel, Pierre R. Marcoux, "Mid-infrared multispectral lensless imaging for wide-field and label-free microbial identification," Proc. SPIE 11359, Biomedical Spectroscopy, Microscopy, and Imaging, 113590B (1 April 2020); doi: 10.1117/12.2557502 


\title{
Mid-infrared multispectral lensless imaging for wide-field and label- free microbial identification
}

\author{
Joël Le galudec ${ }^{\mathrm{a}}$, Mathieu Dupoy ${ }^{\mathrm{a}}$, Véronique Rebuffel ${ }^{\mathrm{a}}$ and Pierre R. Marcoux ${ }^{\mathrm{a}}$ \\ ${ }^{a}$ CEA-LETI MINATECH, 17 avenue des Martyrs, 38054 Grenoble, France
}

\begin{abstract}
Microbial identification is a critical process aiming at identifying the species contained in a biological sample, with applications in healthcare, industry or even national security. Traditionally, this process relies either on MALDI-TOF mass spectroscopy, on biochemical tests and on the observation of the morphology of colonies after growth on a Petri dish. Here is presented an innovative method for label-free optical identification of pathogens, based on the multispectral infrared imaging of colonies. This lensless imaging technique enables a high-throughput analysis and wide-field analysis of agar plates. It could yield very high correct identification rates as it relies on an optical fingerprint gathering both spectroscopic and morphologic features.

The setup consists of a Quantum Cascade Lasers light source and an imager, a square 2.72 by $2.72 \mathrm{~mm}$ uncooled bolometer array. Microorganisms to be analyzed are streaked on a porous growth support compatible with infrared imaging, laid on top of an agar plate for incubation. When imaging is performed, growth support is put in close contact with the imaging sensor and illuminated at different wavelengths. After acquisition, an image descriptor based on spectral and morphological features is extracted for each microbial colony. Supervised classification is finally performed with a Support Vector Machine algorithm and tested with tenfold cross-validation. A first database collecting 1012 multispectral images of colonies belonging to five different species has already been acquired with this system, resulting in a correct identification rate of $92 \%$. For these experiments, multispectral images are acquired at nine different wavelengths, between 5.6 and $8 \mu \mathrm{m}$.

Considering the optimization possibilities of the image descriptors currently used and the ongoing development of the uncooled bolometers technology, these very first results are promising and could be dramatically improved with further experiments. Thereby, mid-infrared multispectral lensless imaging has the potential to become a fast and precise Petri dish analysis technology.
\end{abstract}

Keywords: multispectral, infrared imaging, lensless imaging, microbiology, diagnosis, Support Vector Machine

\section{INTRODUCTION}

Microbiological diagnosis consists in identifying the species present in a sample (bacteria, archaea, fungi ...). This process has many applications in clinical, military and industrial environments and traditionally relies on the observed phenotypic characteristics of microorganisms, such as cell shape, colony morphotype, differential staining, or the results to biochemical testing. These methods are still in use but are gradually replaced by more recent technologies such as mass spectrometry (MALDI-TOF) ${ }^{1}$, molecular biology (PCR) ${ }^{2}$ or Fourier Transform Infrared Spectrometry (FTIR) ${ }^{3}$. These new technologies are generally more expensive, but makes it possible to meet the major challenges of diagnosis: to give more precise, automated and, above all, faster identifications.

However, despite these technical advances, preliminary microbial culture on Petri dish is still often required. This growth step is used to produce a sufficient amount of biological material for further analyses, such as identification by MALDI-TOF or antibiotic susceptibility testing (AST) $)^{4}$, but also to spatially separate the different species present in a polymicrobial sample. Thus, it can be used as a first sorting step to differentiate between "normal" and pathogenic colonies, based on morphological criteria or through the use of chromogenic media ${ }^{5}$. That is why solid plate culture has been the keystone of diagnostic microbiology for the past decades and will likely remain so for years to come ${ }^{6}$.

An instrument capable of performing microbial identification directly from the Petri dish, with neither reagent nor sample preparation, would be a crucial step towards a fully automated identification process. It would also allow, if necessary, to guide the choice for further analyses. Among the technologies capable of rapidly analyzing whole colonies 
on a Petri dish in a nondestructive way, label-free optical methods, such as Raman spectroscopy ${ }^{7}$, elastic light scattering ${ }^{8,9}$ or Fourier-transformed infrared spectroscopy $(\mathrm{FTIR})^{10}$ are of particular interest.

Specifically, infrared spectroscopy is one of the oldest label-free optical identification technologies ${ }^{11}$. It provides insights on the composition of bacteria by analyzing some of the vibrational modes of covalent bonds. Each strain of bacteria has its own specific infrared fingerprint, which allows efficient identification and even subtyping ${ }^{12}$. The midinfrared region is particularly rich in information, with most organic molecules showing their highest absorption peaks between 3 and $8 \mu \mathrm{m}$. Recently, this technology has seen an increasing interest with the emergence of hyperspectral imaging. It consists in acquiring spectra not on the sample as a whole but on several discrete points, creating an image were each pixel corresponds to a unique spectrum. It provides spatially located spectral data and analyses relatively wide areas $^{13}$. This technology provides much more detailed data, but also requires longer acquisition times and rely on complex instrumentation, which limits its applicability as a routine diagnosis method.

Fast microbial identification with simpler instrumentation has recently been performed with another technique, called visible lensless imaging ${ }^{14}$. By removing all optical components and placing the sample almost in contact to the sensor, this technology can rapidly analyzes several colonies with an optical field ranging from several $\mathrm{mm}$ up to a few $\mathrm{cm}$ wide, depending on the sensor. The provided morphological data might be detailed enough to yield species identification ${ }^{15}$, but these results need to be confirmed with much wider databases, including much more species and many strains per species.

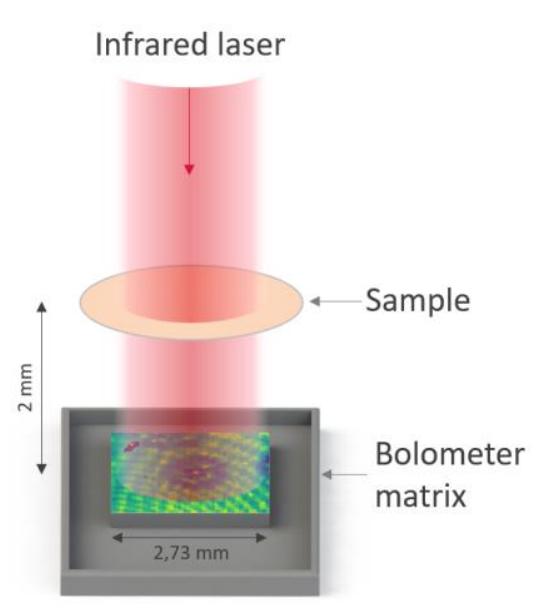

Acquisition

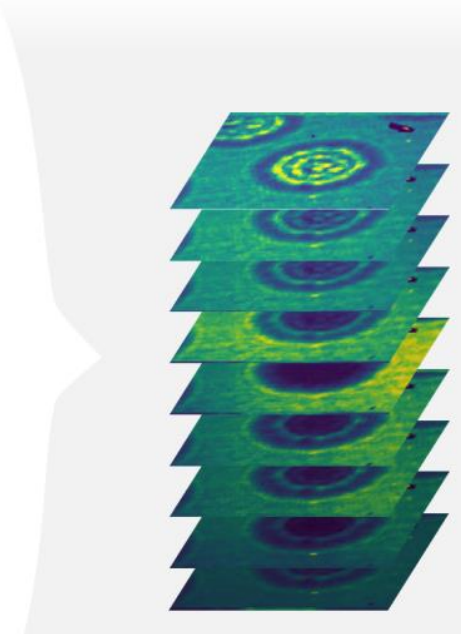

Multispectral stack

\begin{tabular}{|ll|}
\hline C. albicans & $93 \%$ \\
S. epidermidis & $7 \%$ \\
E. coli & $0 \%$ \\
E. cloacae & $0 \%$ \\
\hline
\end{tabular}

Species prediction by Machine learning

Figure 1. Mid-infrared multispectral lensless imaging principle.

Here, we present a combination of these two techniques, called mid-infrared lensless multispectral imaging (Figure 1). It uses a polychromatic infrared light source and a bolometer array to rapidly record transmission images of the sample lighted with a few wavelengths of biological interest (Figure 2). The resulting multispectral images provide both morphological and spectral data covering a field of several $\mathrm{mm}^{2}$, allowing for fast analysis of colonies growing on a Petri dish. This analysis aims at identifying all the bacterial colonies present on a given field of view, down to species, in a fast, nondestructive and label-free way. 


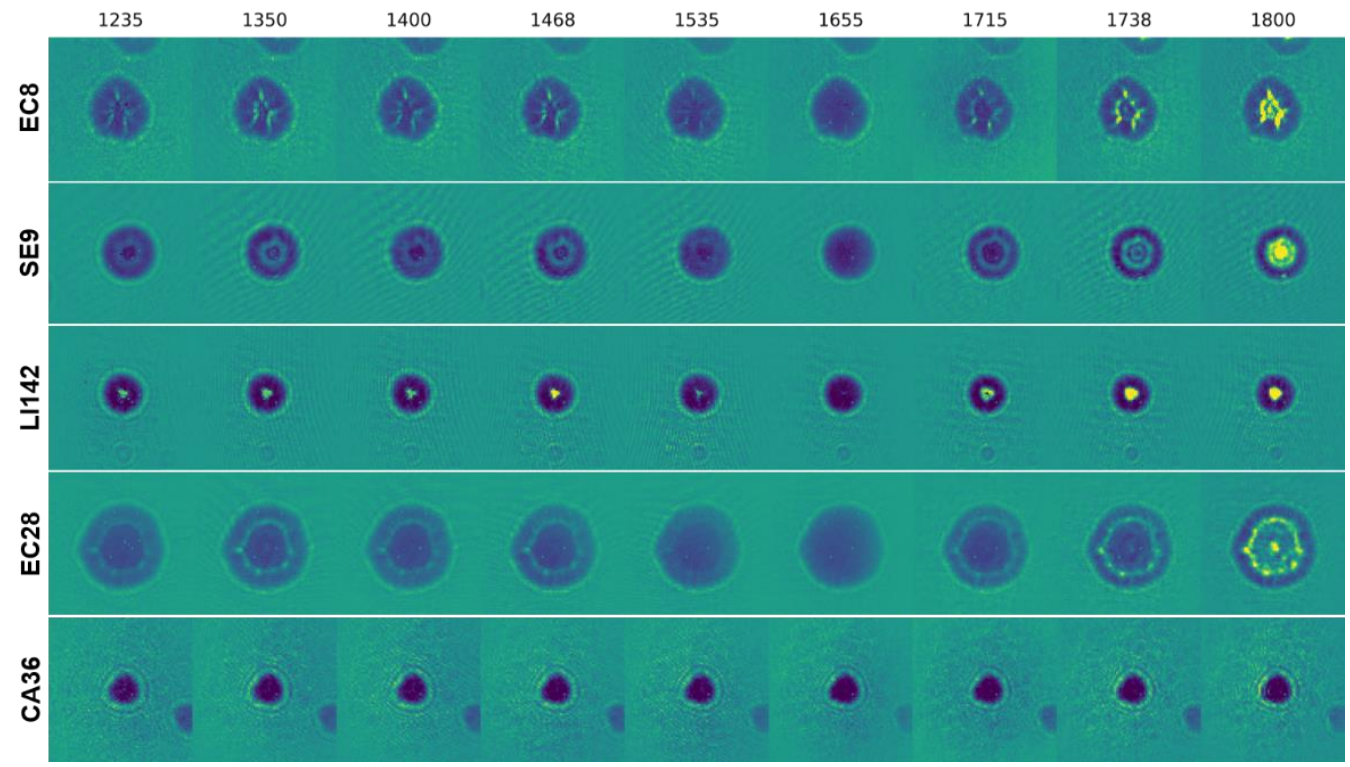

Figure 2. Multispectral images of representative examples from the five species of the database, identified by their short names (described in Table 2). Wavenumbers on top of each column are in $\mathrm{cm}^{-1}$.

\section{MATERIAL AND METHODS}

\subsection{Optical setup}

For this experiment, a Daylight Solutions MIRcat quantum cascade laser (QCL) system is used as a tunable light source. It emits a coherent infrared laser ranging from 5 to $11 \mu \mathrm{m}\left(2000-900 \mathrm{~cm}^{-1}\right)$. The detector is a square $2.73 \mathrm{~mm}$ wide uncooled bolometer array. It uses the pixel-level-packaging technology developed by the CEA-LETI ${ }^{16}$, which currently limits the sensor resolution to $80 * 80$ pixels, but is low cost and allows to bring the sample as close as possible to the bolometers. A programmable microscopy stage enables movements of the sample above the bolometer array with an image-object distance of $2 \mathrm{~mm}$.

Table 1. Wavelengths list.

\begin{tabular}{|lllll|}
\hline & Wavenumber $\left(\mathbf{c m}^{-1}\right)$ & Wavelength $(\boldsymbol{\mu m})$ & Laser power $(\mathbf{m W})$ & Chemical function \\
\cline { 2 - 5 } $\mathbf{2}$ & 1235 & 8.1 & 1.5 & PO \\
$\mathbf{3}$ & 1350 & 7.4 & 10.5 & Reference \\
$\mathbf{4}$ & 1400 & 7.14 & 5.7 & COO- $^{-}$ \\
$\mathbf{5}$ & 1468 & 6.83 & 5.3 & $\mathrm{CH}_{2}$ \\
$\mathbf{6}$ & 1655 & 6.67 & 6.9 & Amide (mode 1) \\
$\mathbf{7}$ & 1715 & 6.05 & 6 & Amide (mode 2) \\
$\mathbf{8}$ & 1738 & 5.8 & 10.7 & COOH \\
$\mathbf{9}$ & 1800 & 5.74 & 18.9 & CO Esters \\
\hline
\end{tabular}

The resulting multispectral stacks are composed of images of a unique zone observed at nine different illumination wavelengths, as described in Table 1. These wavelengths correspond to well-known infrared absorption rays, associated with specific functional groups, and were chosen for their biological relevance ${ }^{9,10}$. Acquisition time for each image is around $20 \mathrm{~ms}$.

One particularity of this system is that the light source is composed of four QCL with specific emission bands and power efficiency. As a result, maximal laser power varies greatly from one wavelength to another. For this first experiment, we choose to keep the laser set to its maximal available power and compensate the lightning variations with the bolometer parameters. This allowed to be sure to have enough signal, regardless of the sample absorption. Now that the system and samples are more known, further experiments will use a laser modulation and not a sensor modulation. 


\subsection{Biological samples}

The method was tested on five different microbial colony-forming species, four bacteria and one yeast, as described in Table 2. These strains were chosen to test the ability of multispectral imaging to distinguish between close species (EC8 and EC28), distant species (LI142 and SE9) and almost non-related species (CA36 and all bacteria).

Table 2. Microbial strain list and database summary. The last two columns show the number of multispectral stacks acquired and the corresponding number of colonies extracted. Several colonies can be present on the same image, especially for species yielding smaller colonies, which makes data acquisition faster for these species.

\begin{tabular}{|c|c|c|c|c|c|}
\hline & Species & Domain & $\begin{array}{l}\text { Strain } \\
\text { reference }\end{array}$ & $\begin{array}{r}\text { Stacks } \\
\text { acquired }\end{array}$ & Colonies \\
\hline CA36 & Candida albicans & Eucarya (yeast) karyote & ATCC 14053 & 173 & 260 \\
\hline EC28 & Escherichia coli & $\begin{array}{l}\text { Bacteria (Gram-negative bacillus, } \\
\text { Enterobacteriaceae) }\end{array}$ & ATCC 11775 & 169 & 171 \\
\hline EC8 & Enterobacter cloacae & $\begin{array}{l}\text { Bacteria (Gram-negative bacillus, } \\
\text { Enterobacteriacea) }\end{array}$ & ATCC 13047 & 139 & 156 \\
\hline LI142 & Listeria innocua & Bacteria (Gram-positive bacillus) & ATCC 33090 & 115 & 158 \\
\hline SE9 & Staphylococcus epidermidis & Bacteria (Gram-positive coccus) & ATCC 14990 & 224 & 267 \\
\hline
\end{tabular}

The main trouble of infrared spectroscopy with biological sample is the strong absorption of water in this range of wavelengths, which normally ban analysis of samples thicker than a few hundred micrometers (here, a whole Petri dish). This problem was overcome by a technique not described here, but allowing normal colony growth and morphology conservation during analysis.

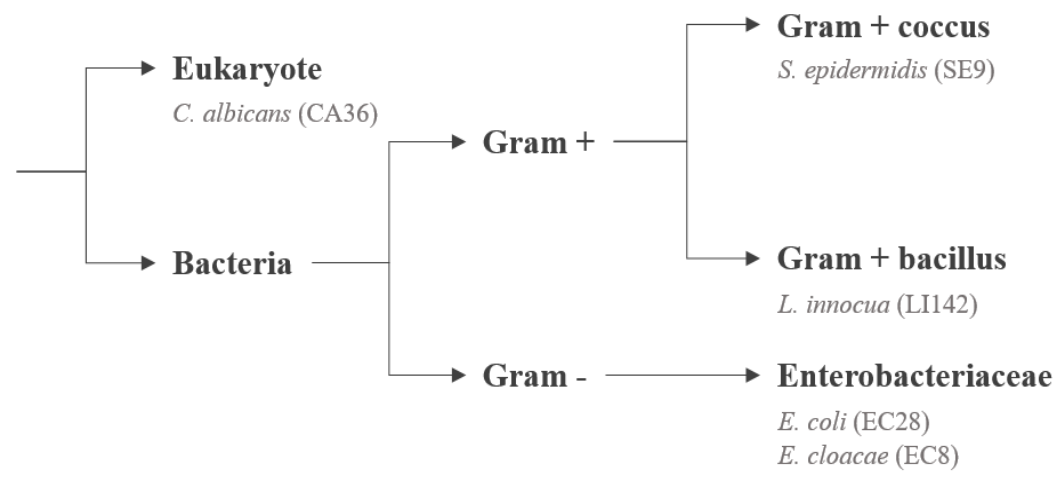

Figure 3. Schematic phylogenetic tree of the selected species.

Microbial species are streaked on a Columbia blood agar solid medium (COS) and incubated for 24 hours at $36.5^{\circ} \mathrm{C}$ before analysis by multispectral lensless infrared imaging.

\subsection{Data processing}

Multispectral stacks are analyzed with custom Python 3.7 scripts, using functions provided by the libraries skimage and sklearn. Before analysis, each image is preprocessed by subtracting a reference background image acquired at the same wavelength and on the same sample on zones clear of microbial growth. Colonies are located from their binary masks on the $1655 \mathrm{~cm}^{-1}$ image, using edge detection algorithm provided by the skimage.feature module. These localization data are then used to extract image descriptors as presented in Table 3 . These parameters are similar to the one used for diagnosis in visible lensless imaging ${ }^{15}$.

To assess the system capability to identify unknown colonies, machine-learning classification is performed by Support Vector Machine (kernel: radial basis function, $\mathrm{c}=10$, gamma $=0.05$ ) with a tenfold cross-validation test. $90 \%$ of the database is used to construct a model, which then predicts a species for the $10 \%$ left. This process is performed ten times, until each colony gets associated with a predicted species. To smooth statistical noise induced by the random database split, full classification is run 30 times. The final predicted species for each colony is then chosen by a majority vote and 
confronted to the real species. Classification results are summarized in a confusion matrix (Figure 4), showing the identification rates for each class.

Table 3. Descriptor parameters.

\begin{tabular}{|llll|}
\hline & Parameter & Wavelength used & Description \\
\cline { 2 - 4 } $\mathbf{2}$ & Radius & 1655 & Radius in pixels \\
$\mathbf{2}$ & Roundness & 1655 & $4 \pi *\left(\right.$ area / perimeter $\left.{ }^{2}\right)$ \\
$\mathbf{3}$ & Skewness & All & Asymmetry of the associated image histogram \\
$\mathbf{4}$ & Texture difference & All & Dissimilarity of the grey-level co-occurrence matrix \\
$\mathbf{5}$ & Transmittance & All & Average transmittance of the whole colony \\
$\mathbf{6}$ & Central intensity & All & Average transmittance of a central disk with $1 / 4$ of the colony radius \\
$\mathbf{7}$ & Donutness & All & Total colony transmittance divided by transmittance of a central disk \\
& & & with $1 / 2$ colony radius \\
\hline
\end{tabular}

\section{RESULTS AND DISCUSSION}

For this proof of concept, a database containing 1012 colonies belonging to five different species was acquired (Table 2). The optical setup and machine learning analysis allowed classifying all species with a correct identification rate (CIR) of at least 92\%, as shown on confusion matrix in Figure 4. The only eukaryote in the base is recognized in 99\% of cases, but more surprisingly, even relatively close species such as E. cloacae (EC8) and E. coli (EC28) are efficiently distinguished, with a confusion rate between these two classes of around $1 \%$. EC8 is the most difficult class to identify $(\mathrm{CIR}=92,7 \%)$, but it is also the one with the lowest number of examples (156). Supplementary analysis tend to show that results are significantly better with at least 200 to 250 colonies per class (data not shown).

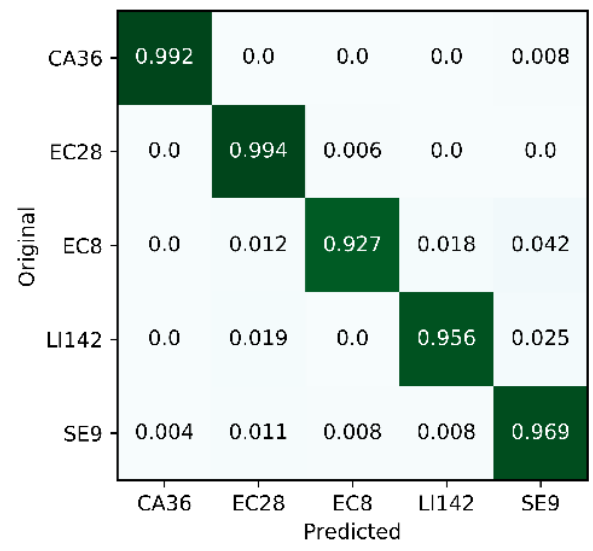

Figure 4. Normalized SVM classification results. Each line of the confusion matrix shows the global identification rate for a given species confronted to all other species.

The main idea of multispectral compared to hyperspectral imaging is to acquire only a restricted set of wavelengths instead of a continuum, which allows faster acquisitions at the price of less detailed data. Here, nine wavelengths were acquired as a first estimate. To measure the impact of the number of wavelengths on classification efficiency, data analysis was run with each possible wavelengths combination. To smooth statistical noise, classifications were performed ten times with each combination. For each set length, the best possible global CRI was kept and summarized in Figure 5. 


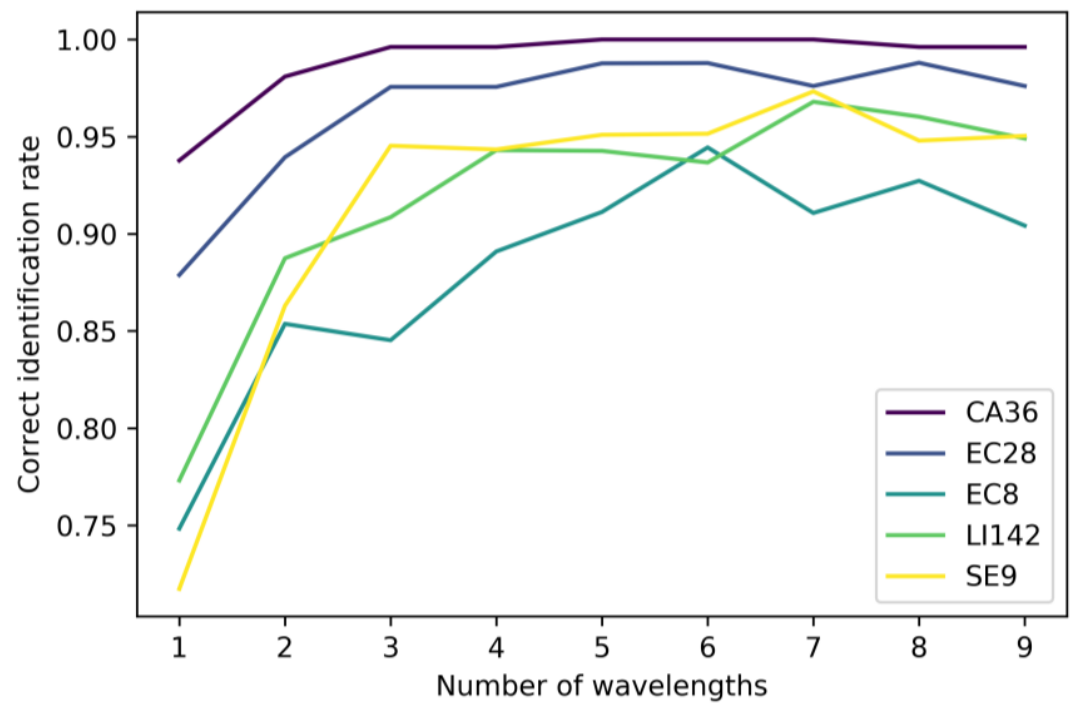

Figure 5. Impact of the number of wavelengths allowed for classification on correct identification results.

As it appears, with our current descriptors and species, using more than five or six wavelengths do not necessarily yields better results. CRI for all species are already above $90 \%$ with five wavelengths and tend to stabilize or even decrease beyond this point. As already discussed, this analysis would need more data, especially for EC8, but it already provides a first estimate of the type of evolution that can be expected for further works. Hereby, it seems that a more restricted set of four to six carefully chosen wavelengths could provide satisfying results. Optimizing the number of wavelengths could enhance CRI but also simplify the instrumentation and contributes to reduce acquisition times.

\section{PERSPECTIVES}

Mid-infrared multispectral lensless imaging is hereby a promising technology for culture-based microbial diagnosis. It offers effective microbial species identification based on whole-colony imaging with a simple setup. However, the whole process can still be greatly improved in several ways. First, larger microbolometer arrays with a higher resolution will provide more detailed images and a larger field of view. The current image descriptors are still basic and have not yet been optimized. Refining the most relevant parameters and wavelengths should improve efficiency and prevent potential overfitting. As observed with this first database, results improve with the number of examples per class, with a minimal acceptable number being around 250 , and not 150 as initially thought.

Several experiments are already planned to push the boundaries of the technology, including its ability to distinguish strains belonging to the same species.

\section{REFERENCES}

[1] Patel, R., "MALDI-TOF MS for the diagnosis of infectious diseases," Clin. Chem. 61(1), 100-111 (2015).

[2] Biofire., "The FilmArray® System," BioFire Diagn., <https://www.biofiredx.com/products/filmarray/> (1 October 2019 ).

[3] Mariey, L., Signolle, J. P., Amiel, C. and Travert, J., "Discrimination, classification, identification of microorganisms using FTIR spectroscopy and chemometrics," Vib. Spectrosc. 26(2), 151-159 (2001).

[4] Lagier, J.-C., Edouard, S., Pagnier, I., Mediannikov, O., Drancourt, M. and Raoult, D., "Current and Past Strategies for Bacterial Culture in Clinical Microbiology," Clin. Microbiol. Rev. 28(1), 208-236 (2015).

[5] Perry, J. D. and Freydière, A. M., "The application of chromogenic media in clinical microbiology," J. Appl. Microbiol. 103(6), 2046-2055 (2007).

[6] Belkum, A. van, Ph.D, Durand, G., M.D, Peyret, M., Ph.D, Chatellier, S., Ph.D, Zambardi, G., Ph.D, Schrenzel, J., M.D, Shortridge, D., Ph.D, Engelhardt, A., Ph.D, Jr, and W. M. D. and Ph.D., "Rapid Clinical Bacteriology and Its Future Impact,” Ann. Lab. Med. 33(1), 14-27 (2013). 
[7] Strola, S. A., Marcoux, P. R., Schultz, E., Perenon, R., Simon, A.-C., Espagnon, I., Allier, C. P. and Dinten, J.-M., "Differentiating the growth phases of single bacteria using Raman spectroscopy," Biomed. Vib. Spectrosc. VI Adv. Res. Ind. 8939, 893905, International Society for Optics and Photonics (2014).

[8] Genuer, V., Gal, O., Méteau, J., Marcoux, P., Schultz, E., Lacot, É., Maurin, M. and Dinten, J.-M., "Optical elastic scattering for early label-free identification of clinical pathogens," Adv. Biomed. Clin. Diagn. Surg. Guid. Syst. XIV 9698, 96980A, International Society for Optics and Photonics (2016).

[9] Marcoux, P., Dupoy, M., Cuer, A., Kodja, J.-L., Lefebvre, A., Licari, F., Louvet, R., Narassiguin, A. and Mallard, F., "Optical forward-scattering for identification of bacteria within microcolonies," Appl. Microbiol. Biotechnol. 98(5), 2243-2254 (2014).

[10] Wenning, M. and Scherer, S., "Identification of microorganisms by FTIR spectroscopy: perspectives and limitations of the method," Appl. Microbiol. Biotechnol. 97(16), 7111-7120 (2013).

[11] Maquelin, K., Kirschner, C., Choo-Smith, L.-P., van den Braak, N., Endtz, H. P., Naumann, D. and Puppels, G. J., "Identification of medically relevant microorganisms by vibrational spectroscopy," J. Microbiol. Methods 51(3), 255-271 (2002).

[12] Johler, S., Stephan, R., Althaus, D., Ehling-Schulz, M. and Grunert, T., "High-resolution subtyping of Staphylococcus aureus strains by means of Fourier-transform infrared spectroscopy," Syst. Appl. Microbiol. 39(3), 189-194 (2016).

[13] Lasch, P., Stämmler, M., Zhang, M., Baranska, M., Bosch, A. and Majzner, K., "FT-IR Hyperspectral Imaging and Artificial Neural Network Analysis for Identification of Pathogenic Bacteria," Anal. Chem. 90(15), 8896-8904 (2018).

[14] Maeda, Y., Dobashi, H., Sugiyama, Y., Saeki, T., Lim, T., Harada, M., Matsunaga, T., Yoshino, T. and Tanaka, T., "Colony fingerprint for discrimination of microbial species based on lensless imaging of microcolonies," PLOS ONE 12(4), B.-L. Lee, Ed., e0174723 (2017).

[15] Maeda, Y., Sugiyama, Y., Kogiso, A., Lim, T.-K., Harada, M., Yoshino, T., Matsunaga, T. and Tanaka, T., "Colony Fingerprint-Based Discrimination of Staphylococcus species with Machine Learning Approaches," Sensors 18(9), 2789 (2018).

[16] Dumont, G., Rabaud, W., Yon, J.-J., Carle, L., Goudon, V., Vialle, C., Becker, S., Hamelin, A. and Arnaud, A., "Current progress on pixel level packaging for uncooled IRFPA," Infrared Technol. Appl. XXXVIII 8353, 83531I, International Society for Optics and Photonics (2012). 